University of Nebraska - Lincoln

DigitalCommons@University of Nebraska - Lincoln

\title{
Microsatellite DNA Analysis Shows that Greater Sage Grouse Leks Are Not Kin Groups
}

\author{
Robert M. Gibson \\ University of Nebraska - Lincoln, rgibson@unl.edu \\ Debra Pires \\ University of California - Los Angeles \\ Kathleen S. Delaney \\ University of California - Los Angeles \\ Robert K. Wayne \\ University of California - Los Angeles
}

Follow this and additional works at: https://digitalcommons.unl.edu/bioscifacpub

Part of the Life Sciences Commons

Gibson, Robert M.; Pires, Debra; Delaney, Kathleen S.; and Wayne, Robert K., "Microsatellite DNA Analysis Shows that Greater Sage Grouse Leks Are Not Kin Groups" (2005). Faculty Publications in the Biological Sciences. 227.

https://digitalcommons.unl.edu/bioscifacpub/227

This Article is brought to you for free and open access by the Papers in the Biological Sciences at DigitalCommons@University of Nebraska - Lincoln. It has been accepted for inclusion in Faculty Publications in the Biological Sciences by an authorized administrator of DigitalCommons@University of Nebraska - Lincoln. 


\title{
Microsatellite DNA Analysis Shows that Greater Sage Grouse Leks Are Not Kin Groups
}

\author{
Robert M. Gibson, ${ }^{\mathrm{a}}$ Debra Pires, ${ }^{\mathrm{b}}$ Kathleen S. Delaney, ${ }^{\mathrm{b}}$ and Robert K. Wayne ${ }^{\mathrm{b}}$
}

aSchool of Biological Sciences, University of Nebraska-Lincoln, Nebraska, USA

bepartment of Ecology and Evolutionary Biology, University of California, Los Angeles, California, USA

Correspondence: Robert M. Gibson, rgibson2@unl.edu

\begin{abstract}
The spectacular social courtship displays of lekking birds are thought to evolve via sexual selection, but this view does not easily explain the participation of many males that apparently fail to mate. One of several proposed solutions to this 'lek skew paradox' is that kin selection favors low-ranking males joining leks to increase the fitness of closely related breeders. We investigated the potential for kin selection to operate in leks of the greater sage grouse, Centrocercus urophasianus, by estimating relatedness between lekking males using microsatellite DNA markers. We also calibrated these estimates using data from known families. Mean relatedness within leks was statistically indistinguishable from zero. We also found no evidence for local clustering of kin during lek display, although males tended to range closer to kin when off the lek. These results make kin selection an unlikely solution to the lek skew paradox in sage grouse. Together with other recent studies, they also raise the question of why kin selection apparently promotes social courtship in some lekking species, but not in others.
\end{abstract}

Keywords: Kinship, Lek skew paradox, Microsatellite analysis, Sage grouse

Received June 27, 2005; revision accepted August 10, 2005

\section{INTRODUCTION}

Leks, clusters of male display territories visited by females for mating, are often interpreted as a product of sexual selection, in which males aggregate to increase mating opportunities (Höglund \& Alatalo 1995). However, this view does not easily explain the participation of many males that apparently fail to mate, a problem dubbed the 'lek skew paradox' by Kokko (1997). Multiple, nonexclusive hypotheses have been proposed to resolve this difficulty. First, males might lack sufficient information to optimize lek-settlement decisions, for example because female copying behavior makes individual mating success unpredictable(Kokko 1997). As a result males might stay at leks where their reproductive prospects were poor. Second, unsuccessful males might be queuing for future breeding status (Wiley 1973; McDonald \& Potts 1994; Kokko et al. 1998). Third, nonreproductive benefits, e.g. reduced predation risk, may be sufficient to maintain lek membership even if immediate breeding prospects are poor (Lack 1968; Trail 1987; Gibson et al. 2002; Boyko et al. 2004). Finally, the participation of low-ranked males might increase the fitness of close relatives in the same group and therefore be a product of kin selection (Kokko \& Lindström 1996).
Interest in the last possibility has been stimulated by both prospective theoretical modelling (Kokko \& Lindström 1996) and molecular genetic data suggesting that males of several lekking bird species display with kin (Höglund et al. 1998a; Petrie et al. 1999; Bouzat \& Johnson 2003). These studies suggest the potential for kin selection to operate in avian leks, but fall short of demonstrating that low-ranking males have higher inclusive fitness when displaying with kin than under alternative scenarios, e.g. displaying alone or with unrelated companions. The latter has been shown for wild turkeys in which coalitions of related males court females on the run rather than defending lek territories (Krakauer 2005). Kin selection is apparently not relevant to some other lekking and quasi-lekking species in which males do not display with or near relatives (McDonald \& Potts 1994; Martin et al. 2001; Madden et al. 2004). Its proposed role in white-bearded manakin leks (Shorey et al. 2000) also appears uncertain in light of very low mean within-lek relatedness reported by Höglund \& Shorey (2003).

The greater sage grouse (Centrocercus urophasianus) (henceforth sage grouse) is a large, lek-breeding bird native to sagebrush shrub steppe in western North America. Males display on leks at dawn each day during early spring in groups that can range in size from < 
5 to $>100$ individuals. Afterwards, they forage and rest in sagebrush habitats nearby, often returning to the lek at dusk to display or roost. A lek is attended daily by males that defend display territories and, less regularly, by other nonterritorial males that may attend a single lek or range more widely. Females visit leks on 2-3 days annually, and mate on their last visit. Breeding is relatively synchronized between females, with most copulations occurring over a 10-day period (Bradbury et al. 1989). Within leks, mating distributions are usually highly skewed (Wiley 1973; Semple et al. 2002) and variation in male mating success has been linked to female choice based on multiple criteria, including male acoustic display performance, mating site fidelity and, particularly, the choices of other females ('copying') (Gibson et al. 1991).

Our previous field studies of this species in eastern California have provided little evidence that low-ranking males are engaged in reproductive queuing (Gibson et al. 1991; Gibson 1992), but have implicated dilution of predation risk as a benefit of lek display (Boyko et al. 2004).

Here we consider whether kinship might also be relevant to resolving the lek skew paradox in this system. A rigorous confirmation of this hypothesis would require showing both that males display with kin and that this behavior increases the inclusive fitness of lowranking males relative to alternatives, such as displaying either with nonrelatives or alone. However, an inclusive fitness analysis becomes relevant only if males display with kin. Here we combine microsatellite DNA analysis and behavioral data to investigate whether this is the case.

\section{MATERIALS AND METHODS}

We studied the lekking behavior of a resident population of greater sage grouse in Long Valley, California, from 1984 to 2001 . This study analyses data collected in two periods (1984-1988 and 1997-2001, except 1999) and at three major leks (LV2, 4 and 8) of the seven to nine that were active annually (Gibson 1996, Figure 1a).

We trapped birds for marking and blood sampling by spotlighting and cannon netting at one to three major leks annually. Additional birds were trapped in winter flocks or during the summer. All males were marked with coloured lek bands for individual recognition and, to monitor ranging behavior off the lek, we also fitted some with small, tail-mounted radio transmitters. Blood for DNA extraction was collected from an over-clipped hallux nail and stored either as a thin film smear fixed in methanol (1984-1988) or in Queens College lysis buffer (Seutin et al. 1991) (1997-2001). We obtained useable DNA from 262 adults, including 181 males trapped and/or observed at leks in 16 lek-years (LV2: 89 males/4 years; LV4: 74/9; LV8: 18/3). Additional DNA samples were obtained from the broods of 10 females (Semple et al. 2002).

\section{Behavioral methods}

We systematically recorded the lek attendance, location and agonistic behavior of colour-banded individuals during daily, or near daily observations, of the dawn lek using previously described methods (Gibson et al. 1991). We classified males as territorial if they consistently occupied the same area from which they aggressively excluded other males. To measure an individual's proximity to other territorial males at the same lek, we used the mean $X$ and mean $Y$ coordinates of locations mapped during scan and/or focal animal samples (mean $\pm \mathrm{SD}=48 \pm 40$ points per male, $n=47$ ). We determined territorial status and location for banded males at one focal lek in each sampled year from 1984 to 2000, and territorial status, but not location, for males at all three major leks in 2001. At some leks we did not determine territorial status of some or all banded males trapped or observed, either because the lek was not the focus of intensive observations or because individuals did not attend often enough for their status to be determined. Consequently, our analyses deal with two types of sample: (i) all males captured or observed at a lek in a given year, regardless of territorial status and (ii) the subset of marked individuals known to be territorial. As noted above (see Introduction), territorial males exhibit a stronger affinity for a single lek site. Hence, if kin associate it should be more easily detectable in the latter subset.

To measure male associations off the lek we located radio-tagged individuals daily by triangulation after the morning lek when they were foraging or resting away from the lek. We measured dyadic spatial associations by the mean daily distance between individuals, and estimated the degree to which males with overlapping ranges moved together from day to day using an index of association based on the second preference index of Jacobs (1974). This index takes values from minus one (mutual avoidance) through zero (independent movement) to plus one (completely coordinated movement). Association measures were computed using the dynamic interaction module in the program RANGES6 (Anatrack Ltd). We analysed ranging data for males tracked on at least 10 days (mean $\pm \mathrm{SD}=22.6 \pm$ $11.7 \mathrm{~d}, n=30$ ) in lek-years with > five males sampled (LV2 in 1988, LV4 in 2000, and LV2 in 2001).

\section{Molecular analysis}

We isolated total genomic DNA from blood samples 
in buffer or blood scraped from prepared slides using a DNeasy kit (QIAGEN). Genomic DNA from slides was concentrated in $50 \mu \mathrm{L}$ of elution buffer, instead of $200 \mu \mathrm{L}$ as recommended by the protocol. We used three microsatellite markers originally cloned in red grouse: LLSD3, LLSD8 and LLST1 (Piertney \& Dallas 1997; Piertney et al. 1998) and four from the capercaillie: TUT1, TUT2, TUT3 and TUD3 (Segelbacher et al. 2000). In addition, we isolated four species-specific loci using a biotinylated oligo probe for the following motifs: AACC, AAGG and AAAG (Bardeleben et al. 2004). The variable loci used were CUAACC8b (F primer 5'-TGAAATCATCTTCGGGGAAA-3', R primer $5^{\prime}$-CCTAAAGAATTATCCTATTGACGA-3'), CUAAGG37 (F primer 5'-GGCCTTCTAAAGTTATGCAGTTTT-3', R primer 5'-CTTCATCTTCACGGAGCACA-3'), CUAACC46a (F primer 5'-ATCCCTGCCTACCTGAAATC-3', R primer 5'-AGCCAAAATCTCTCCCTCAT-3') and CUAAAG43 (F primer 5'-GGCAAAATAGAAGTTATGAAGTG-3', $\mathrm{R}$ primer $5^{\prime}$-TAAAGCCACATCGCTGGAAT-3'). For CUAAGG37, TUD3, LLST1, LLSD5 and LLSD8, the appropriate DNA fragments were amplified using polymerase chain reaction (PCR) in $25-\mu \mathrm{L}$ reaction volumes containing 1-3 $\mu \mathrm{L}$ of DNA extract, $10 \mathrm{~mm}$ Tris- $\mathrm{HCl}(\mathrm{pH}$ 9.0), $50 \mathrm{~mm} \mathrm{KCl}, 0.1 \%$ Triton $X-100$, and $2.5 \mathrm{~mm} \mathrm{MgCl}_{2}$ and $200 \mu \mathrm{m}$ of each dNTP. All other primers used reaction mixtures with $1.5 \mathrm{~mm} \mathrm{MgCl}$. One unit of Taq DNA polymerase (Sigma) and 10 pmoles of primer were used in each reaction mix. The PCR amplification profile is as follows: an initial denaturation at $94^{\circ} \mathrm{C}$ for $3 \mathrm{~min}$, then 25-28 cycles of $94^{\circ} \mathrm{C}$ for $30 \mathrm{~s}, 30 \mathrm{~s}$ annealing and $72^{\circ} \mathrm{C}$ for $40 \mathrm{~s}$ extension. A 5 -min extension step at $72^{\circ} \mathrm{C}$ was the final step after all cycles were finished. Annealing temperatures were as follows for each locus: $56^{\circ} \mathrm{C}$ for TUT1, 2, 3, TUD3, CUAACC 8 b; $55^{\circ} \mathrm{C}$ for CUAAGG37, CUAAAG43, CUAACC $46 a ; 50^{\circ} \mathrm{C}$ for LLST1 and LLSD8; and a touchdown $60-50^{\circ} \mathrm{C}$ for LLSD3.

We genotyped individuals at all 11 loci using fluorescently labelled forward primers and unlabelled reverse primers. Dye-labelled primers were created with Beckman Coulter dyes D2, D3 or D4. Genotypes were scored on the Beckman Coulter CEQ200XL DNA analysis system (Fullerton). When suitable, PCR products were pooled for multiple loci of the same individual. All individuals were typed twice to ensure correct genotype assignment, and samples from slides were genotyped a third time for confirmation.

\section{Statistical analysis}

Screening genetic markers. We tested each locus for consistency with Hardy-Weinberg equilibrium (HWE), investigated changes in allele frequencies over time and tested for linkage disequilibrium between loci using genepop 3.3 (Raymond \& Rousset
1995). Our sample spans two 5-year periods separated by 9 years (1984-1998 and 1997-2001). Because 5 years is shorter than maximum lifespan whereas 9 years is long enough for complete population turnover, we tested for changes in allele frequencies between but not within periods. Allele frequencies differed significantly between sample periods at 6 of 11 loci. Therefore subsequent genetic computations were carried out separately for each period.

There was no statistical evidence of linkage disequilibrium between any pair of loci in either period. However, there were significant heterozygote deficits at 7 of the 11 loci in period one and at 2 of the same loci in period two (Table 1). At 3 of the 7 loci with significant heterozygote deficits, family data revealed null alleles, inferred from the combination of a homozygous mother with one or more offspring homozygous for a paternal allele. To assess the possible influence of null alleles on our results we performed all analyses with and without the three affected loci. Our main conclusions were unchanged. Consequently we report the analyses using the full 11-locus panel, thereby retaining maximal genetic resolution. However, we state in the text where results obtained using the 8-locus panel differed.

Testing kin associations. We used two approaches to investigate whether males attending the same lek were more related than expected by chance. First, we computed mean coefficients of relatedness $(r)$ for samples of males within lek-years using the program RELATEDNESS 5.0.8 (Queller \& Goodnight 1989) and compared the sample means to a null expectation of zero using a $t$-test. Population allele frequencies were based on all putatively unrelated birds sampled in the same period, excluding the focal lek. To ensure statistical independence, we limited the sample to 8 of the 16 sampled lek-years that did not share overlapping dyads (LV2: 2 years; LV4: 4; LV8: 2). We sampled $16.4 \pm 11.8$ (mean \pm SD) males per lek-year (range 7-36) representing $44 \pm$ $25 \%$ (range $23-90 \%$ ) of the mean numbers of males attending per day (50 \pm 46 , range 10-125). To calibrate these estimates we used the same method to estimate relatedness within families, specifically between mothers and offspring and among brood mates. Note that relatedness makes two estimates of $r$ for the former relationship (from mother to offspring and from offspring to mother). Second, for all sampled lek-years we generated dyadic estimates of relatedness, tested them for evidence of close genetic relationship $(r=0.5$ vs. a null hypothesis of $r=0$ ) using the program kinship 1.3.1 (Queller \& Goodnight 1989), and then compared numbers of dyads in which the null hypothesis was rejected with the number expected from the type 1 error rate for the test. Where the same dyad occurred 
Table 1 Characteristics of 11 microsatellite loci used in this study

\begin{tabular}{lccccccccc} 
& \multicolumn{3}{c}{ Period 1: $1984-1988(N=163)$} & \multicolumn{3}{c}{ Period 2: 1997-2001 $(N=99)$} \\
Locus & Alleles & $H_{O}$ & $H_{E}$ & HWE & Alleles & $H_{O}$ & $H_{E}$ & HWE \\
CUAAAG43 & 10 & 0.600 & 0.803 & $*$ & 11 & 0.532 & 0.799 & $*$ \\
CUAACC8b & 2 & 0.218 & 0.234 & & 2 & 0.288 & 0.324 \\
CUAAGG37 & 10 & 0.490 & 0.747 & $*$ & 10 & 0.732 & 0.813 \\
CUAAGG46a & 20 & 0.586 & 0.792 & $*$ & 16 & 0.820 & 0.857 \\
LLSD3 & 8 & 0.529 & 0.637 & $*$ & 7 & 0.602 & 0.656 \\
LLSD8 & 9 & 0.641 & 0.648 & $*$ & 6 & 0.696 & 0.673 \\
LLST1 & 2 & 0.484 & 0.496 & $*$ & 2 & 0.577 & 0.488 \\
TUD3 & 11 & 0.303 & 0.514 & $*$ & 11 & 0.357 & 0.672 & $*$ \\
TUT1 & 5 & 0.507 & 0.727 & $*$ & 4 & 0.571 & 0.709 \\
TUT2 & 2 & 0.355 & 0.354 & & 2 & 0.321 & 0.317 \\
TUT3 & 4 & 0.665 & 0.692 & & 4 & 0.643 & 0.697
\end{tabular}

$H_{O^{\prime}}$ observed heterozygosity; $H_{E^{\prime}}$ expected heterozygosity; ${ }^{*} H_{O}$ deviates significantly from Hardy-Weinberg equilibrium after Bonferroni adjustment. $H_{O}$ and $H_{E}$ values computed using cervus 2.0 (Marshall et al. 1998).

in different samples it was counted only on the first occurrence. We were unable to extend the dyadic approach to the detection of more distant relatives due to loss of statistical power. For example, power averaged 0.89 (range across lek-years $=0.76-0.93$ ) for detection of full-sibs or parent-offspring dyads $(r=0.5)$, but fell to 0.47 (range 0.39-0.57) for half-sibs $(r=0.25)$. However, the proportion of dyads that were identified as putative kin was similar in the two cases, suggesting that the detected frequency of close kin may be representative of more distant genetic relationships as well.

Sage grouse leks are often large enough to contain multiple clusters of male territories. In light of both the suggestion that leks might be spatially divided into kin groups (Shorey et al. 2000) and our dyadic test results (see below), we also investigated associations between dyadic estimates of relatedness and spatial proximity within leks using Mantel tests. We analysed this issue in two contexts: on display territories in the early morning and away from the lek later in the day. As with estimation of mean relatedness, we analysed data only from leks whose dyadic membership was independent of other samples. We used one-tailed probabilities to test the directional hypothesis that kin associate spatially, but all Mantel tests involving other variables are two-tailed (see Results).

\section{RESULTS}

\section{Mean relatedness within families}

Mean relatedness estimates within families were statistically indistinguishable from the expected value of 0.5 between mother and offspring and among full sibs (mother- offspring: mean $\pm \mathrm{SD}=0.491 \pm 0.133$; offspring-mother: $0.508 \pm 0.136$; within brood: $0.467 \pm$ 0.127 ; one sample $t$-tests: $P=0.433-0.853$ ), indicating that our estimates of relatedness are unbiased (Figure 1).

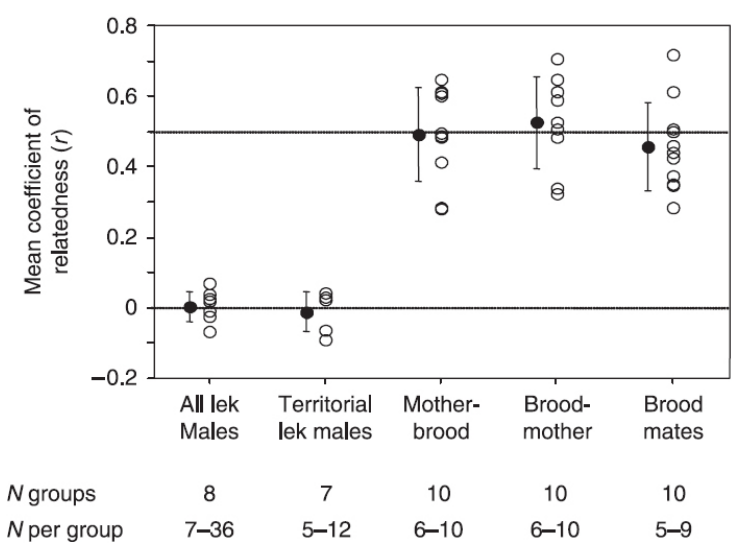

Figure 1 Mean coefficients of relatedness within leks and families. Values for groups are shown as open circles and the mean for each type of group as filled circles \pm SD.

\section{Mean relatedness within leks}

Mean coefficients of relatedness within leks were close to (and statistically indistinguishable from) zero, whether calculated for all males sampled (across lek mean $\pm \mathrm{SD}=0.003 \pm 0.043$; one-sample $t=0.195$, d.f. $=$ $7, P=0.851$ ) or just known territorial birds (mean $\pm \mathrm{SD}$ $=-0.012 \pm 0.057$; one-sample $t=0.571$, d.f. $=6, P=0.589$ ) (Figure 1). Mean relatedness values for both samples were also significantly lower than relatedness estimates within families (unpaired $t$-tests: $P<0.0001$ ).

\section{Kin clustering within leks?}

Although on average leks were assemblages of unrelated males, dyadic tests revealed pairs of closely related birds at slightly above random levels. Across all sampled lek-years, likelihood tests rejected the null hypothesis of $r=0$ in favor of $r=0.5$ in $7.5 \%$ (161) of 2152 dyads involving 181 individuals, or $2.5 \%$ more than expected by chance. When the analysis was restricted to known territorial males, $7 \%$ (20) of 293 dyads involving 
Table 2 Mantel correlations between territorial proximity and relatedness

$\begin{array}{lccl}\text { Lek-year } & \text { Males } & \text { Mantel } r & \text { 1-tailed } P \\ \text { LV2-1988 } & 12 & 0.145 & 0.859 \\ \text { LV4-1984 } & 10 & -0.173 & 0.114 \\ \text { LV4-1987 } & 11 & 0.026 & 0.570 \\ \text { LV4-1997 } & 5 & 0.207 & 0.721 \\ \text { LV4-2000 } & 9 & 0.100 & 0.727\end{array}$

63 individuals were significant, or $2 \%$ more than expected by chance.

Tables 2 and 3 summarize Mantel correlations between dyadic relatedness and spatial proximity of males both on lek territories and when off the lek. Relatedness was not significantly correlated with territory proximity in any of the five samples and only one was in the direction predicted if kin display in close proximity. Thus territorial males did not associate spatially with kin when displaying.

In contrast, kin tended to range closer together than unrelated males as indicated by negative correlations between relatedness and proximity in all three off-lek samples (Table 3). The pattern is significant in two of three samples, and all three were significant when relatedness was estimated omitting the three loci with null alleles. To test whether this pattern represented active social affiliation between male kin we computed correlations between Jacobs' association index, which measures coordinated movement (see Methods), and both relatedness and proximity (Table 3 ). Proximity was negatively correlated with Jacobs' index in all three samples and significantly in two, indicating that males that spent more time near each other were more likely to range together. However, relatedness was not significantly associated with Jacobs' index in any sample, suggesting that kin did not actively associate. Instead, range proximity among kin might be explained by common influences during range establishment.

Consistent with their differing patterns of covariation with relatedness, on- and off-lek proximity were not significantly correlated with each other within the two samples for which data were available (Mantel tests, LV2-1988: Mantel $r=0.010, n=5, P=0.795$; LV4-2000: $r=-0.015, n=7, P=0.946)$. However, due to small sample sizes, this conclusion should be accepted cautiously.

\section{DisCUSSION}

Our data suggest that sage grouse leks are largely assemblages of unrelated males, with little if any spatial association between kin either within or between leks. The only exception occurred away from the lek, but even here we found little evidence of active social affiliation between relatives. The absence of kin association during lek display implies that kin selection is unlikely to resolve the lek skew paradox in sage grouse and therefore that the solution must lie elsewhere.

This conclusion could be incorrect if our samples were unrepresentative, if we underestimated mean relatedness, or if acceptance of null hypotheses reflected low statistical power rather than a lack of kin structure. However, none of these potential criticisms seems plausible. On average our lek samples approached half the mean daily attendance (see Methods), in some cases representing almost complete coverage of territorial males. While a single sample might not be representative, we analysed multiple samples and found consistently low mean relatedness within leks. Possible underestimation of mean relatedness is contradicted by family data, which yielded accurate estimates of the expected coefficients of relatedness. The possibility of insufficient statistical power is also not persuasive. As shown above, tests for the presence of close kin within leks had relatively high power. Additionally, because we detected spatial association between kin when birds were off the lek, low power is an unlikely explanation for its absence when males were displaying.

If kin selection does not resolve the lek skew paradox in this case, what does? As noted in the Introduction, our previous analyses suggest that dilution of predation risk can explain the participation of low-ranking males (Boyko et al. 2004), but that queuing is unlikely to provide a solution (Gibson 1992). Kokko's (1997) suggestion that low-ranking males participate because individual mating success is unpredictable also seems unlikely in light of evidence both (i) that individual mating success is statistically repeatable within and between breeding seasons (Gibson, unpublished) and (ii) that males exhibit a win-stay/lose-shift pattern of territory occupancy that should result in individuals abandoning sites where they are unsuccessful (Gibson 1992). However, it is possible that inertia in the process of territory settlement and resettlement plays

\begin{tabular}{lcllllll}
\multicolumn{2}{l}{ Table 3 Mantel correlations between off-lek proximity, relatedness $(r)$ and Jacobs' index of association (I) (see Methods) } \\
Lek-year & Males & Mantel $r$ & 1-tailed $P$ & Mantel $r$ & 2-tailed $P$ & Mantel $r$ & 2-tailed $P$ \\
LV2-1988 & 11 & -0.207 & 0.064 & -0.457 & $0.0008^{*}$ & 0.110 & 0.438 \\
LV2-2001 & 11 & -0.280 & $0.0128^{*}$ & -0.729 & $0.0001^{*}$ & 0.177 & 0.155 \\
LV4-2000 & 8 & -0.494 & $0.008^{*}$ & -0.311 & 0.197 & 0.218 & 0.269
\end{tabular}

*Significant after sequential Bonferroni adjustment. 
a role. For example, some unsuccessful males initially display persistently before moving on, while some individuals exhibit strong fidelity to a lek where they have mated previously even if they subsequently fail to attract females.

This study and other recent analyses of kinship in lekking birds have a produced a heterogeneous set of outcomes. It is now clear that kin selection explains the participation of low-ranking males in at least one case (Krakauer 2005), that it might play a role in at least three additional species (Höglund et al. 1998a; Petrie et al. 1999; Bouzat \& Johnson 2003), but is unlikely to do so in several others (McDonald \& Potts 1994; Martin et al. 2001; this study). These results suggest three implications for future studies of this topic. First, because current evidence indicates that multiple factors can promote the participation of low-ranking males in leks, progress in understanding this phenomenon will require investigations that address the full range of possibilities. Second, if such investigations determine that male kin associate, it will be important to also investigate whether low-ranking male have higher inclusive fitness when displaying with kin vs. displaying alone or with nonrelatives. This requires estimates of (i) mean within-group relatedness and (ii) the effects of an individual's lek membership on both its own fitness and that of other group members. Finally, because kin selection appears to promote social display in some species but not in others, it will be important to identify the conditions that cause this variation. We will explore the last topic elsewhere.

In conclusion, our data indicate that male greater sage grouse do not display with relatives, and hence that factors other than kin selection must resolve the lek skew paradox in this species. This outcome emphasizes the importance of considering multiple hypotheses when attempting to understand the roles of low-ranking males in avian leks. Together with a heterogeneous set of results from other recent studies of this topic, it also raises the question of why kin selection appears to play a role in some lekking species but not in others.

\section{Acknowledgements}

This project was supported by the NSF (principally IBN-0078013) with additional funding from the Departments of Biology at UCLA and University of Nebraska-Lincoln. Of over 50 individuals who assisted with fieldwork, the following made particularly important contributions: S. Balenger, J. Hale, K. Kapp, J. Meunier, G. Sadoti, D. Shapiro, E. Spotswood, J. Thompson, and L. Wolfenbarger. We thank the US Bureau of Land
Management, USDA Forest Service and Los Angeles Department of Water and Power for permission to work on their land, the UC Natural Reserve System for logistical support, and G. Bachman, D. Pilson, K. Reeve and S. Shuster for useful discussions. This study was conducted under permits from the California Department of Fish and Game and with the approval of the UCLA and UN-L Animal Care and Use Committees.

\section{References}

Bardeleben, C., V. Palchevskiy, R. Calsbeek, and R.K. Wayne (2004). Isolation of polymorphic tetranucleotide microsatellite markers for the brown anole ( $\mathrm{An}$ olis sagrei). Molecular Ecology Notes 4: 176-178.

Bouzat, J.L., and K. Johnson (2003). Genetic structure among closely spaced leks in a peripheral population of lesser prairie chickens. Molecular Ecology 13: 499505.

Boyko, A.R., R.M. Gibson, and J.R. Lucas (2004). How predation risk affects the temporal dynamics of avian leks: greater sage grouse versus golden eagles. American Naturalist 163: 154-165.

Bradbury, J.W., R.M. Gibson, C.E. McCarthy, and S.L. Vehrencamp (1989). Dispersion of displaying male sage grouse. II. The role of female dispersion. Behavioral Ecology and Sociobiology 24: 15-24.

Gibson, R.M. (1992) Lek formation in sage grouse: the effect of female choice on male territory settlement. Animal Behavior 43: 443-450.

Gibson, R.M. (1996). A re-evaluation of hotspot settlement in lekking sage grouse. Animal Behavior 52: 9931,005 .

Gibson, R.M., J.W. Bradbury, and S.L. Vehrencamp (1991). Mate choice in leking sage grouse revisited: the roles of vocal display, female site fidelity and copying. Behavioral Ecology 2: 165-180.

Gibson, R.M., A.S. Aspbury, and L. McDaniel (2002). Active formation of mixed-species grouse leks: a role for predation in lek evolution? Proceedings of the Royal Society of London. Series B, Biological Sciences 269: 2,5032,508 .

Höglund, J., and R.V. Alatalo (1995). Leks. Princeton University Press, Princeton, New Jersey.

Höglund, J., and L. Shorey (2003). Local genetic structure in a white-bearded manakin population. Molecular Ecology 12: 2,457-2,463.

Höglund, J., R.V. Alatalo, A. Lundberg, P.T. Rintamaki, and J. Lindell (1998). Microsatellite markers reveal the potential for kin selection in black grouse leks. Proceedings of the Royal Society of London. Series B, Biological Sciences 266: 813-816.

Jacobs, J. (1974). Quantitative measurement of food selection. A modification of the forage ratio and Ivlev's electivity index. Oecologia 14: 413-417. 
Kokko, H. (1997). The lekking game: can female choice explain aggregated male displays? Journal of Theoretical Biology 187: 57-64.

Kokko, H., and J. Lindström (1996). Kin selection and the evolution of leks: whose success do young males maximize? Proceedings of the Royal Society of London. Series B, Biological Sciences 263: 919-923.

Kokko, H., J. Lindstrom, R.V. Alatalo, and P.T. Rintamaki (1998). Queuing for territory positions in lekking black grouse (Tetrao tetrix). Behavioral Ecology 9: 376-383.

Krakauer, A. (2005). Kin selection and cooperative courtship in wild turkeys. Nature 434: 69-72.

Lack, D. (1968). Ecological Adaptations for Breeding in Birds. Methuen, London.

Madden, J.R., T.J. Lowe, H.V. Fuller, et al. (2004). Neighbouring male spotted bowerbirds are not related, but do maraud each other. Animal Behavior 68: 751-758.

Marshall, T.C., J. Slate, L.E.B. Kruuk, and J.M. Pemberton (1998). Statistical confidence for likelihood-based paternity inference in natural populations. Molecular Ecology 7: 639-655.

Martin, C.A., J.C. Alonso, J. Alonso, C. Pitra, and D. Lieckfeldt (2001). Great bustard population structure in central Spain: concordant results from genetic analysis and dispersal study. Proceedings of the Royal Society of London. Series B, Biological Sciences 269: 119-125.

McDonald, D.B., and W.K. Potts (1994). Cooperative display and relatedness among males in a lek-mating bird. Science 266: 1,030-1,032.

Petrie, N., A. Krupa, and T. Burke (1999). Peacocks leks with relatives even in the absence of social and environmental cues. Nature 401: 155-157.

Piertney, S.B., and J.F. Dallas (1997). Isolation and characterization of hypervariable microsatellites in the red grouse (Lagopus lagopus scotticus). Molecular Ecology 6: 93-95.

Piertney, S.B., A.D.C. MacColl, P.J. Bacon, and J.F. Dallas (1998). Local genetic structure in red grouse (Lago- pus lagopus scotticus): evidence from microsatellite DNA markers. Molecular Ecology 7: 1,645-1,654.

Queller, D.C., and K. Goodnight (1989). Estimating relatedness using genetic markers. Evolution 43: 258275.

Raymond, M., and F. Rousset (1995). genepop (version 1.2): a population genetics software for exact tests and ecumenicism. Journal of Heredity 86: 248-249.

Segelbacher, G., I.J. Paxton, G. Steinbruck, P. Trontelj, and I. Storch (2000). Characterization of microsatellites in the capercaillie, Tetrao urogallus (Aves). Molecular Ecology 9: 1,934-1,935.

Semple, K.E., R.K. Wayne, and R.M. Gibson (2002). Microsatellite analysis of female mating behavior in lek-breeding sage grouse. Molecular Ecology 10: 2,0432,048 .

Seutin, G., B.N. White, and P.T. Boag (1991). Preservation of avian blood and tissue samples for DNA analysis. Canadian Journal of Zoology 69: 82-90.

Shorey, L., S. Piertney, J. Stone, and J. Hoglund (2000). Fine-scale genetic structuring on Manacus manacus leks. Nature 408: 352-453.

Trail, P.W. (1987). Predation and antipredator behavior at Guianian cock-of-the-rock leks. Auk 104: 496-507.

Wiley, R.H. (1973). Territoriality and non-random mating in sage grouse Centrocercus urophasianus. Animal Behavior Monographs 6: 85-169.

Robert Gibson studies behavioral, ecological and evolutionary processes underlying social behavior, with a focus on lek mating behavior in birds. Debra Pires has studied the phylogeography of African bats and is currently investigating biogeography and genetic differentiation of rodents on the coastal islands of British Columbia. Katy Semple Delaney is a postdoc with UCLA and the National Park Service, studying finescale genetic structure of vertebrate populations in habitats fragmented by urbanization. Robert Wayne applies molecular genetic techniques to questions in animal ecology, evolution and behavior. 